\title{
ABPI. Aprendizaje Basado en Proyectos Integrados y acción tutorial del profesorado. Una innovación metodológica global en la formación de estudiantes de Magisterio.
}

Javier Herrero-Martín ${ }^{a}$, Rosario Valdivielso Alba ${ }^{a}$, David Fonseca Escudero ${ }^{\text {b }}$ Xavier Canaleta Llampallas $^{\mathrm{c}}$

\footnotetext{
a Departamento de Educación Infantil y Primaria. Facultad de Educación. Centro Superior de Estudios Universitarios La Salle (Universidad Autónoma de Madrid). Madrid, Spain. i.herrero@lasallecampus.es; r.valdivielso@lasallecampus.es.

${ }^{b}$ Departamento de Arquitectura. GRETEL- Group of Research on Technology Enhanced Learning. La Salle/Univerdiad Ramón Lllull. Barcelona. Spain. David.fonseca@1asalle.url.edu

'Departamento de Ingeniería. GRETEL-Group of Research on Technology Enhanded Learning. La Salle/Universidad Ramón Llull. Barcelona, Spain. xavier.canaleta@salle.url.edu
}

\section{\$EWWFW}

$\square$

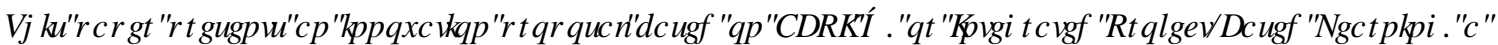

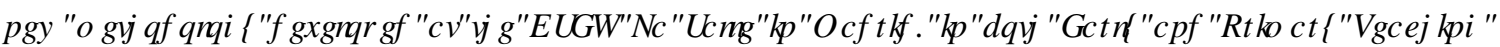

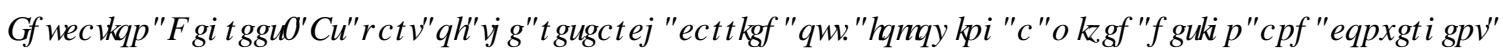

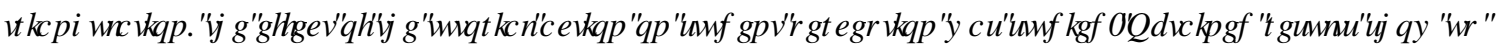

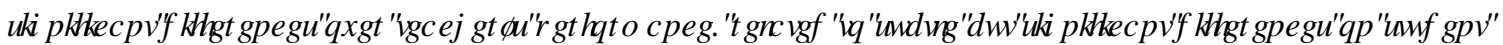

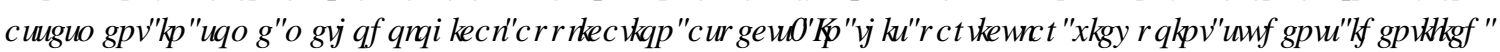

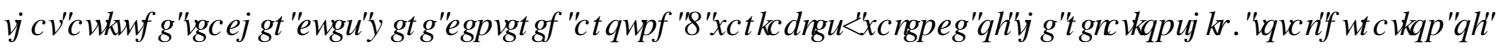

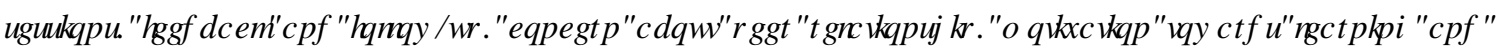

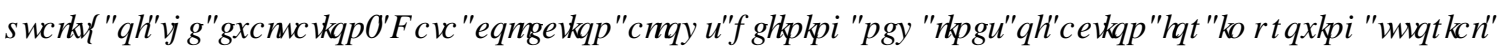

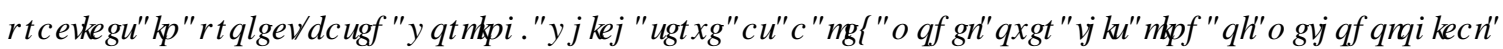
FKDQJ HP DNQJ $\square S L R F H M H D$

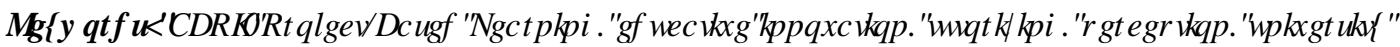
ODQTQT

\section{HXP HQ}

]

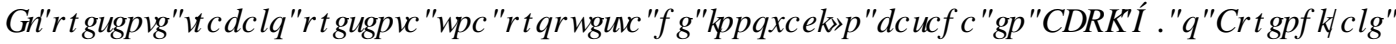

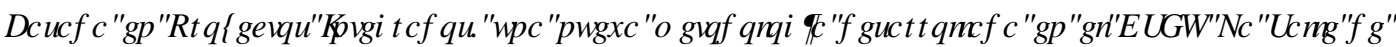

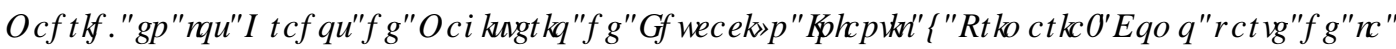

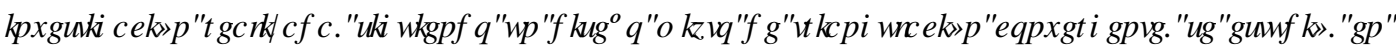

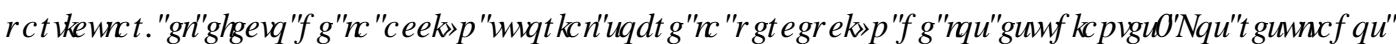

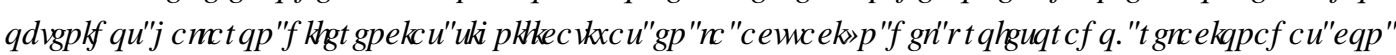

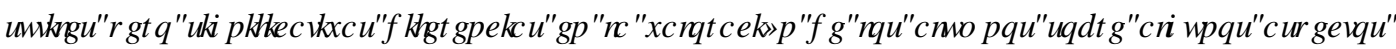

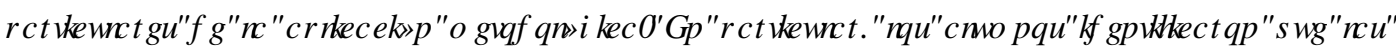




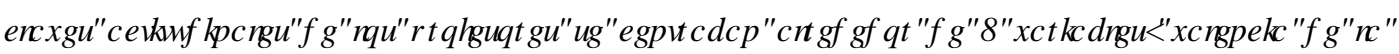

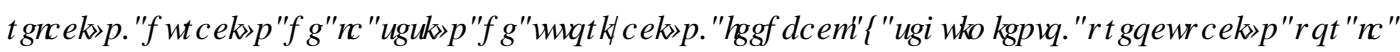

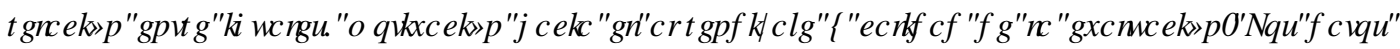

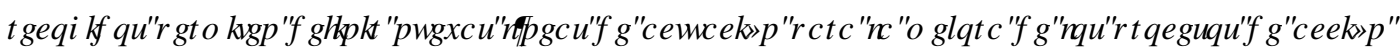

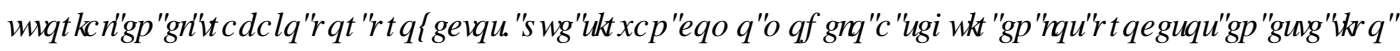
GHWDQMRLP DFIRQHIP HRGRQ JIFDVI

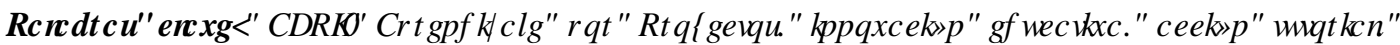

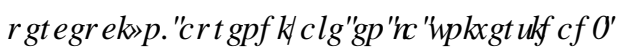

\section{Introducción}

La inclusión de cambios en el diseño de propuestas metodológicas ocupa en nuestros días un foco sustancial en el repertorio de propuestas de innovación educativa en nuestro país. En concreto, la posibilidad de trascender un modelo de corte tradicional, basado en la acción magistral y en la participación pasiva del alumno, parece haber dado paso a una nueva y más reciente visión relacionada con el trabajo colaborativo y cooperativo. En este contexto, el llamado Aprendizaje Basado en Proyectos (ABP) ha venido a ocupar un lugar relevante en este escenario de actividad, posibilitando que la formación universitaria incorpore dinámicas de trabajo colaborativo (Zamora et al., 2018) sobre la base de la organización de proyectos focales, generalmente dependiente de asignaturas o grupos concretos de asignaturas, más o menos relacionadas entre sí (Toledo y Sánchez, 2018; Alonso-Ferreiro, 2018).

Aprender a través de proyectos facilita el abordaje interdisciplinar del conocimiento (Olivares, 2018). Por esta razón, en los últimos años está siendo crecientemente aplicado a diversas áreas de formación universitaria (McLeod y van der Veen, 2019; Pérez-Aranda et al., 2015)) y, en particular, al desarrollo de diferentes propuestas de Grado en Educación, afrontando el proceso de cambio desde la reorganización curricular (Arandía y Fernández, 2012) o de transformación metodológica, dentro de los planes de estudio (Morales y García, 2018).

Probablemente, de todas estas experiencias diversas, la acometida actualmente por el Centro Superior de Estudios Universitarios La Salle ocupa, a nuestro juicio, un papel de especial referencia, por su carácter pionero en la integración global del currículo, tanto transversal como verticalmente, en una parte significativa del Plan de Estudios en la formación de estudiantes de Magisterio en los Grados de Infantil y Primaria, lo que ha supuesto un gran reto innovador relacionado con la transformación metodológica de las enseñanzas en su conjunto. Se presentan a continuación algunos elementos característicos de la propuesta como marco para el análisis de la importancia de la acción tutorial en el aprendizaje basado en proyectos.

Además, y no menos significativo, ABPI se enmarca, como desarrollo metodológico, en el entorno del Nuevo Contexto de Aprendizaje (NCA), una propuesta global, de corte pedagógcio, metodológico y evaluativo, que ha sido diseñada como marco general para la implantación de un modelo común, a nivel nacional de acción, participación y evaluación educativa en los Centros La Salle. De ello se derivan, tanto la presente experiencia sobre ABPI en la universidad La Salle, en el Campus Madrid, como las correspondientes actuaciones en la capa universitaria de NCA, en el Campus La Salle de Barcelona. 


\section{Objetivos}

La presente comunicación tiene por objetivo presentar la experiencia de diseño e innovación metodológica ABPI (C) (Aprendizaje Basado en Proyectos Integrados), describir las líneas genéricas de la estructura general y analizar el impacto de la acción tutorial, considerada como un elemento estructural emergente del propio diseño, sobre la percepción y motivación de los estudiantes.

\section{Desarrollo de la innovación}

Los antecedentes de la propuesta se enmarcan en dos condiciones importantes, necesarias para comprender la realidad que nos ha llevado hasta este momento. Por un lado, la necesidad de que, en el contexto de una Facultad de Educación, cumpliendo con el reconocimiento en los planos ontológico y epistemológico de lo que representa, se propongan nuevas posibilidades que, desde el sustrato científico e investigador, arrojen luz sobre la mejora de los procesos de enseñanza y aprendizaje.

En segundo lugar, ABPI se enmarca en un entorno más amplio de innovación pedagógica, denominado NCA, acrónimo de Nuevo Contexto de Aprendizaje, que está siendo desarrollado desde 2018 como una respuesta de la escuela La Salle a los retos educativos del siglo XXI. Con ello, el proceso de transformación global se dirige a todos los centros escolares de la institución en España y Portugal, y a todas las etapas de escolarización. En este sentido, el desarrollo de NCA ocupa desde la propuesta de transformación de la escuela Infantil, la escuela Primaria, Secundaria, Bachillerato, Ciclos Formativos, Obras Socioeducativas y la Universidad (Campus la Salle en Madrid y Barcelona).

Desde 2017 la Facultad de Educación del Centro Superior Universitario La Salle (en adelante CSEU La Salle) acoge una experiencia NCA que ha sido bautizada como ABPI.

\subsection{Características básicas del modelo ABPI.}

Describimos a continuación algunas condiciones estructurales que ayudarán a comprender inicialmente las características del diseño de innovación propuesto.

- Se dirige a los estudiantes de los Grados de Magisterio de Educación Infantil y Educación Primaria.

- Es una propuesta metodológica de integración transversal del conocimiento.

- Se integran todas las asignaturas del currículo, teniendo en cuenta la organización del Plan de Estudios y las asignaturas correspondientes en cada Grado.

- Se caracteriza por integrar también las asignaturas de 3 WFWFXP, de acuerdo con su distribución en los diferentes cursos.

- Compromete a todo el profesorado. Visibiliza la labor docente, tanto para los estudiantes como para los propios profesores y comunidad universitaria.

- Incorpora, además, una metodología de evaluación propia, que considera tanto la adquisición de conocimientos como la experiencia de organización, gestión y relación, en la actividad universitaria. 


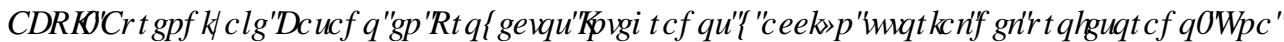

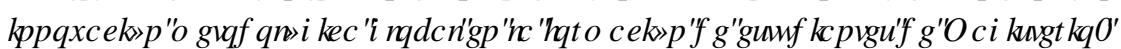

\subsection{Estructura metodológica de ABPI}

ABPI se estructura en dos bloques metodológicos de actividad: a) IDMHP RGXQU $U$ en la que se fundamentan los conocimientos básicos de cada asignatura del Plan de Estudios, y b) IDUHGHSLR| HFIRV, donde aplican e integran el conocimiento alrededor de propuestas narrativas que conducen a los equipos de desarrollo por las diferentes actividades y tareas. La visión general de ABPI permite que, desde el inicio de asignatura, los alumnos proyectan buena parte de lo que van adquiriendo hacia la integración en proyectos.

La actividad en proyectos se desarrolla alrededor de un plan de propuestas narrativas que guían a los equipos en el transcurso de su actividad. El argumentario central de los proyectos narrativos de primer curso gira alrededor de los diversos agentes que integran el acto educativo y los de segundo curso, de las cualidades pedagógicas que debe tener la escuela actual. De este modo, cada proyecto regula, aproximadamente, tres semanas de duración académica y posibilitan el desarrollo de tareas de los equipos, basándose en los siguientes principios:

a) ( OWDEDWDXWQRP R I IDXWRUHXXOR. Los equipos de ABPI son soberanos en su planificación y secuenciación de actividad. Constituidos por 5-7 personas, cada grupo organiza tiempos de actividad, define objetivos intermedios, asigna funciones, responsabilidades y roles (delimitados por la propuesta propia en ABPI) y controla, en primera instancia, el seguimiento de la actividad.

b) ( $Q P R G H \mathbb{R}$ GHDFFly $Q$ WLRUR. Se trata de una estructura que emerge de la propia dinámica metodológica dispuesta en el diseño. Existen tres niveles de tutorización. En primer lugar, el pivote fundamental del sistema es el tutor de Equipo ABPI. Cada profesor de asignatura lidera un Equipo ABPI al que acompaña a lo largo de la actividad de un semestre. Su acción tiene, fundamentalmente un carácter mediador. En segundo lugar, el tutor especialista, más cercano a la figura del profesor de asignatura. Es realizado por los profesores del curso o por otros que actúan de forma complementaria, dado su ámbito de conocimiento específico (por ejemplo, música, arte o matemáticas). En tercer lugar, el tutor de aula, que realiza, por un lado, las tareas tradicionales de esta representación y, por otro, coordina la activad general de los tutores de Equipo ABPI. Todo ello dispone de un sistema de andamiaje que facilita la representación de la práctica en ABPI como un sistema de descubrimiento y participación guiada.

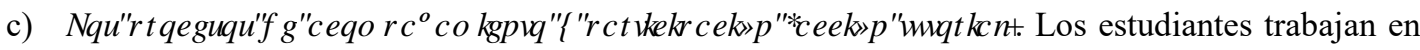
las aulas de proyecto, atendiendo a la planificación semanal que establecen. Sin embargo, con carácter periódico, se realizan encuentros de seguimiento entre ellos y los profesores, siguiendo el plan establecido para ello. Además, cada equipo se encuentra, en algún momento de la semana, con su tutor y se establecen seminarios de seguimiento. Lo mismo sucede con los seminarios de cierre de proyecto (al finalizar cada tramo) o de valoración final (al finalizar el semestre).

d) / DWURWFIRQH. Un elemento esencial para el desarrollo de competencias de trabajo en equipo lo constituyen los cambios estructurales entre los miembros constituyentes. Así, las personas rotan sus roles cada proyecto y rotan en los equipos cada semestre. De este modo, al finalizar sus estudios de Grado, se asegura que todos los estudiantes hayan formado equipo de trabajo con todas las demás personas del aula.

e) ( OSLRUWP DIGHP HQRUUDFly A A partir de segundo curso, cada equipo de desarrollo de proyecto tiene un rol asignado a la mentorización de los equipos del curso inmediatamente inferior. Este rol se ocupa de actuar de referente y guía en la actividad del equipo mentorizado. El modelo así planteado establece que, junto con el sistema de acción tutorial, los propios estudiantes se comprometan con la propia acción pedagógica en el seno de ABPI. 
f) / D $G R F X P$ HQWFIy Q $G H D S R \mid R$. El eje central metodológico es el Mapa de Proyectos, un conjunto de narrativas que contienen todo lo necesario para describir cada proceso de actividad. A partir de ellas, los equipos disponen, además, de material de apoyo sobre FDPSXV YLWDO que complementa el contenido de cada una de las actividades de proyectos. Por otro lado, existe un espacio documental, en el que se encuentran todos los procedimientos, documentos de información de roles, documentos de planificación y seguimiento de actividad y documentos de funciones y responsabilidades.

g) / D IRUP DFIY Q WDQMHWDO Paralelamente al curso de la actividad de Proyectos, tienen lugar talleres y seminarios de formación avanzada, que nutren la actividad, relacionados con algunas competencias fundamentales para los estudiantes (por ejemplo, arte y presentación estética y ecológica, o encuentros interprofesionales alrededor de los proyectos.

h) / DHHDODFIy QLQUUJ $W D$ El proceso de evaluación sigue una metodología propia. Reside en tres factores: a) la evaluación entre iguales (roles y logros); b) la evaluación de seguimiento (portafolios y seguimiento de tutorización), y la evaluación de cierres de proyecto (productos y habilidades de comunicación). Además, la producción correspondiente es evaluada, en su contenido, por cada profesor de asignatura. Con todo, el resultado de la evaluación de cada proyecto considera dos componentes: procesos de gestión y relación y conocimientos desarrollados y adquiridos.

\subsection{Despliegue del programa ABPI.}

En julio de 2017 se creó un Grupo de Innovación Docente (GID-La Salle), con la intención de reflexionar sobre nuevas actuaciones que, desde la innovación, permitiesen avanzar hacia una forma más activa de desarrollar la formación de Maestros. Fruto del trabajo de diseño en esta primera fase, se concretó que la implantación (despliegue) del mismo sería gradual, pues conlleva el desarrollo de guías de proyecto y documentación adicional, por un lado, como la formación y trabajo colaborativo por parte del cuadro de profesores.

En septiembre del curso académico 2018-2019 se desplegó ABPI entre los estudiantes de primer curso de ambos Grados, y en el actual, 2019-2020 nos encontramos desarrollando el programa también en segundo curso. De esta forma, el sistema de seguimiento y garantía de calidad del programa conlleva tres procesos básicos: a) construcción de proyectos de semestre en curso; b) actualización de proyectos del año anterior, en función de los indicadores de evaluación y c) diseño de estructura de proyectos para el año siguiente.

En el momento de presentar esta comunicación, se encuentran trabajando, en paralelo, cuatro grupos de profesores, correspondientes a los desarrollos de proyecto de segundo semestre en el Centro, más otros dos que comienzan a definir la organización de ABPI para el curso próximo.

\subsection{Evaluación del impacto. Metodología de investigación.}

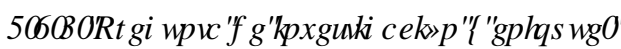

La cuestión fundamental que se plantea como problema de investigación es la siguiente ¿en qué medida el despliegue metodológico de ABPI supone una mejora en los procesos formativos (en cuanto a los procesos de aprendizaje y sus resultados) de los estudiantes de Magisterio de Educación Infantil y Primaria? Y, más concretamente, ¿cómo influye la acción tutorial en la percepción de los estudiantes en el proceso de transformación metodológica?

(cc) EY-NG-ND 2021, Universitat Politècnica de València

CRQJUHR, Q15 HGस००स० 


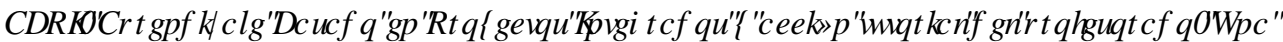

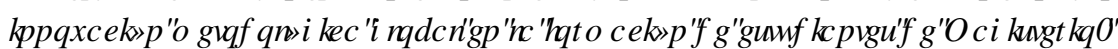

$\square$

Tres fueron los ámbitos focales de estudio: motivación de alumnos. acción tutorial y resultados académicos. Por motivación entendemos el grado de apreciación de la metodología por parte de los estudiantes; por acción tutorial, el grado de implicación en el proceso de actividad de proyectos por parte de los profesores tutores y el rendimiento fue considerado en término de evaluación de resultados, tanto en el nivel de habilidades de grupo como en el de conocimientos.

\section{पष口ण' LMKR}

El diseño de investigación se ha planteado al amparo del paradigma de la complejidad (Morrison, 2002; Cochran-Smith et al., 2014), un marco adecuado para poder analizar cómo los sistemas educativos se desarrollan y se transforman (Martin y McQuitty, 2019). Además, es una alternativa reciente a los paradigmas positivista, interpretativo o crítico, que rompe con el carácter lineal de un proceso investigador en educación para considerar cómo un conjunto de variables han de ser interpretadas en un contexto holístico (Santonus, 1998), pues si se atomiza el análisis focalizado en cada variable de forma independiente, se pierde el valor de la interacción dinámica entre todas las partes de un sistema. La teoría de la complejidad sugiere que las diversas unidades de análisis, en el sistema, interaccionan a todos los niveles, individuo, institución y comunidad (Lemke, 2001).

Desde un punto de vista operativo, la teoría de la complejidad sugiere el uso de procedimientos de investigación acción, metodologías de caso o procesos participativos de investigación, algo que, dada la exigencia de un proceso de implementación metodológica que afecta a la comunidad universitaria en conjunto, se propone como más apropiado pues permite reconocer una diversidad múltiple de perspectivas, causalidades y efectos. Así, investigamos "desde dentro" del propio proceso de acción. Consideramos, en este contexto, al profesor como investigador

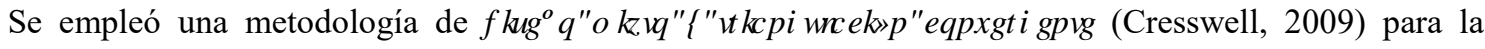
recogida de datos, definiendo dos capas de estudio, una de carácter cuantitativo y otra de tipo cualitativo, que se combinan en una tercera fase de cada ciclo de investigación y acción para extraer y validar adecuadamente los resultados combinados. Se utilizó una aproximación a través de cuestionarios, para recoger una visión general, y un análisis más pormenorizado mediante el uso de entrevistas individuales y grupos focales (grupos de discusión).

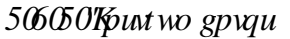

Se construyó un cuestionario amplio constituido por tres escalas diferentes y un apartado de cuestión abierta, para poder recoger datos sobre percepción y motivación de los estudiantes que participaron en el programa ABPI. Los datos recogidos permitieron comparar resultados por grupos de interés (primer o segundo curso o Grado universitario) y establecer agrupaciones utilizando factores de bloqueo (percepción del profesorado y seguimiento de actividad).

Se expresa la necesidad de caminar, dados los beneficios, hacia un modelo que permita diseñar sistemas de integración del conocimiento en la universidad, mediante el uso de recursos disponibles, metodológicos, tecnológicos y organizacionales, con el objetivo de recoger datos sobre aspectos de la cultura del Centro, tales como valores, asunciones, creencias, deseos o problemas experimentados.

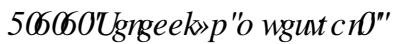

En el desarrollo del programa participaron todos los estudiantes matriculados en la modalidad presencial en los Grados de Educación Infantil y Primaria, en los dos primeros cursos de despliegue. Del total de 157 alumnos matriculados, 122 participantes cumplimentaron los cuestionarios que contenían las escalas y la entrevista semiestructurada ( $\mathrm{IC}=5$; nivel de confianza, 95\%), constituyendo la muestra representativa 
inicial. De ellos, 3 casos fueron eliminados por defectos de cumplimentación de datos (casos perdidos o patrones anómalos de respuesta).

\subsubsection{Adquisición de datos.}

Para la recogida de datos de análisis cuantitativo se utilizaron escalas de construcción ad hoc, dispuestas en un cuestionario online ( 0 IFLRLIW) $R L P$ V), para cada uno de los bloques o estratos de análisis (grupoaula), que fueron analizados, tanto para identificar su estructura final (validación y fiabilidad de escalas) como para su estudio, con el programa estadístico IBM SPSS, v 24.

Para el análisis cualitativo se empleó el programa MAXQDA ( $\$ Q D D U F \bigvee 3 L R$ ), con objeto de facilitar el proceso de etiquetado con códigos de clasificación de categorías y representación de modelos de relación de variables. Previamente, se utilizaron los seminarios semanales de portavoces como grupos de discusión para el análisis. De este modo, se organizaron un total de 6 sesiones por curso, tres por proyecto ABPI, y 30 sesiones de aproximadamente una hora de duración en total. Cada grupo de discusión estuvo conformado por al menos un integrante de cada equipo y varios profesores. Con ello, el tamaño aproximado de los grupos de discusión oscilaba entre 5 y 10 alumnos y 3 a 5 profesores.

\section{Resultados.}

\subsection{Percepción e impacto de la acción tutorial en un diseño de Aprendizaje Basado en Proyectos Integrados.}

En la investigación presentada se utilizó una de las escalas del cuestionario, denominada "percepción de la acción tutorial". Se aplicó un procedimiento factorial de reducción de dimensiones por el que, de los 11 ítems iniciales, la escala final quedó reducida a 9 ítems, que arrojaron un KMO de 0,928 ( $\mathrm{p}=0,000$; Barlet $=817,551)$. Extrayéndose un único componente capaz de explicar un $65,439 \%$ de la varianza, con un índice de fiabilidad (alpha de Cormbach) de 0,932.

Para el estudio de resultados fue aplicado un $P R G H \times C Q H D J H Q H D O P$ XQWDUDQW Se aplicó un ANOVA (15) X (5) X (2), considerando como factores a los equipos tutorizados por el conjunto de profesores que intervinieron en el programa, los grupos de estudio (bloques o estratos) y los niveles relativos a cada curso de estudios, con objeto de observar posibles efectos derivados de la experiencia de trabajo en ABPI. Los resultados más relevantes pudieron apreciarse en el nivel de tutorización de equipos (Tabla 1), si bien se observó un cierto efecto relativo a la interacción entre acción tutorial y curso $(p=0,012 ; 5 D][P D R U G H$ $5 R \mid)$.

(

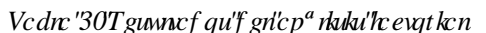

Pruebas multivariante ${ }^{\mathrm{a}}$

\begin{tabular}{lll|l|l|l|l}
\multicolumn{2}{c}{ Efecto } & Valor & F & Gl de hipótesis & $\begin{array}{c}\text { gl de } \\
\text { error }\end{array}$ & Sig. \\
\hline Intersección & Traza de Pillai &, 963 & $241,670^{\mathrm{b}}$ & 9,000 & 84,000 &, 000 \\
\hline Lambda de Wilks &, 037 & $241,670^{\mathrm{b}}$ & 9,000 & 84,000 &, 000 \\
\hline Traza de Hotelling & 25,893 & $241,670^{\mathrm{b}}$ & 9,000 & 84,000 &, 000 \\
\hline Raíz mayor de Roy & 25,893 & $241,670^{\mathrm{b}}$ & 9,000 & 84,000 &, 000 \\
\hline
\end{tabular}

(cc) EY-NC-ND 2021, Universitat Politècnica de València

CRQJ UHRR, Q15 HGस००स० 


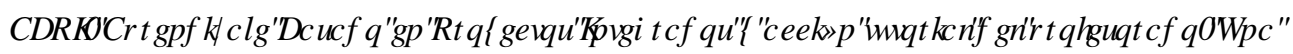

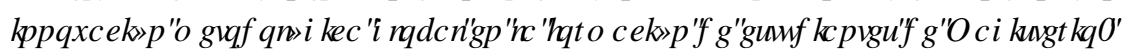

\begin{tabular}{lllllll}
\hline EQABPI & Traza de Pillai & 1,974 & 1,521 & 153,000 & 828,000 &, 000 \\
\cline { 2 - 7 } & Lambda de Wilks &, 085 & 1,619 & 153,000 & 689,236 &, 000 \\
\hline Traza de Hotelling & 3,185 & 1,712 & 153,000 & 740,000 &, 000 \\
\hline Raíz mayor de Roy & 1,199 & $6,491^{\mathrm{c}}$ & 17,000 & 92,000 &, 000 \\
\hline Traza de Pillai &, 493 & 1,360 & 36,000 & 348,000 &, 087 \\
\hline Lambda de Wilks &, 584 & 1,359 & 36,000 & 316,524 &, 089 \\
\hline Traza de Hotelling &, 590 & 1,352 & 36,000 & 330,000 &, 092 \\
\hline & Raíz mayor de Roy &, 264 & $2,549^{c}$ & 9,000 & 87,000 &, 012 \\
\hline
\end{tabular}

a. Diseño: Intersección + EQABPI + CURSO + GRADO + EQABPI * CURSO + EQABPI * GRADO + CURSO * GRADO + EQABPI $*$ CURSO * GRADO

b. Estadístico exacto

c. El estadístico es un límite superior en F que genera un límite inferior en el nivel de significación.

No se observaron efectos en el resto de contrastes, tanto para los niveles de la variable CURSO como de GRUPO.

Respecto al análisis cuantitativo, la actividad resultante de los 30 grupos focales reveló, de forma particular, la importancia del factor tutorial en el seguimiento de la actividad. Particularmente, los estudiantes otorgaban una mayor importancia a este hecho en segundo curso, pues lo situaban en relación con su propia experiencia de actividad en ABPI. La extracción de categorías asociadas a la variable "percepción de la acción tutorial" fue similar a la que constituyó la estructura de la escala de apreciación. Se extrajeron 6 indicadores: YDOQFID GH QD UHDFlyQ (positiva/negativa), GXWFly Q GH DD VHYQ GH

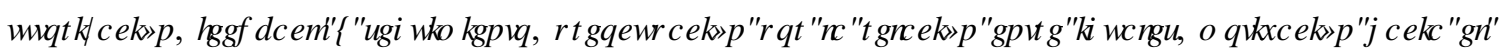
DSLHQD]DNH y FDOCDOF GH DI HDDDFIyQ En síntesis, los resultados apuntaron hacia diferencias importantes entre los tutores de mayor dedicación a los estudiantes y aquellos que dedicaron menos tiempo. Los primeros tendían a ajustarse a los criterios generales establecidos por la coordinación del programa. Los estudiantes reconocen muy positivamente su actividad de acompañamiento, son conscientes del tiempo dedicado por sus tutores en las tareas de seguimiento, tanto con alumnos como con respecto a los documentos y tareas, reciben, con frecuencia, IHHEDFN incluso en momentos que se salen de los tiempos ordinarios (docentes), encuentran que el apoyo en la gestión del conflicto es un pilar fundamental para su actividad (particularmente en primer curso), son con frecuencia apoyados y animados por ellos y encuentran que les evalúan con justicia y adecuación a los criterios establecidos. Sin embargo, los tutores que no se ajustan (o lo hacen deficitariamente) a los criterios normativos establecidos, son considerados como poco interesados, pues dedican un tiempo menor del propuesto como estándar, no ofrecen IHLEDFNadecuado, en tiempo y forma, muestran escasa preocupación por la relación entre iguales, mantienen distancia respecto a la vivencia de los alumnos y tienden a ser percibidos como injustos a la hora de evaluar.

\subsection{Efectos de la acción tutorial sobre variables de motivación.}

Partiendo de la situación anterior de investigación, el análisis se orientó hacia la identificación de posibles efectos de la diversidad de patrones de atención tutorial sobre la dinámica de actividad en ABPI. Para ello, se extrajeron los promedios obtenidos por cada equipo tutorizado en la escala de "percepción de la 
acción tutorial”. A continuación, estas puntuaciones fueron agrupadas alrededor de la nueva variable independiente. Como medidas de la variable dependiente se utilizaron los datos obtenidos en las escalas sobre P RUMDFly Qy pHFHFFly QP HRRRQJIFD, aplicadas a los participantes en el estudio.

Se practicó un análisis de regresión curvilíneo que exploró modelos lineal, cuadrático y logarítmico. Los resultados permitieron establecer una serie de efectos ligeros pero significativos $(p<0,005)$, entre la calidad de la acción tutorial y la acogida de la metodología de evaluación $\left(\mathrm{R}^{2}=0,93 ; \mathrm{p}=0,004\right)$. Algo similar ocurre con el sentido de justicia en la evaluación individual $\left(R^{2}=0,090 ; p=0,004\right)$, la adecuación temático-narrativa de los proyectos $\left(\mathrm{R}^{2}=0,072 ; \mathrm{p}=0,003\right)$, la comprensión del planteamiento evaluativo del sistema $\left(R^{2}=0,065 ; p=0,20\right)$, el tiempo destinado a la realización de proyectos $\left(R^{2}=0,33 ; p=0,48\right)$ o la distribución de los productos a lo largo de los proyectos $\left(\mathrm{R}^{2}=0,029 ; 0,062\right)$.

El análisis cualitativo complementario devolvió, en este punto, un resultado convergente. Los estudiantes

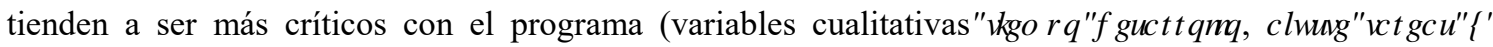

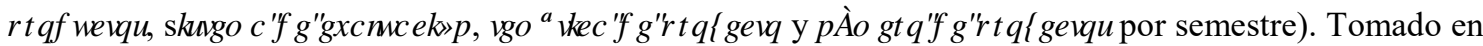
conjunto, los resultados apuntan hacia la necesidad de homogeneizar el proceso de acción tutorial en los procesos de aprendizaje por proyectos en la universidad, particularmente en lo que concierne a la comprensión conjunta y compartida de la metodología de aprendizaje por proyectos.

\section{Conclusiones}

La universidad necesita reconsiderar la forma en que enseña, aprovechando el conocimiento y la investigación que giran alrededor de las llamadas pedagogías activas en educación. En concreto, el aprendizaje basado en proyectos es una opción que se ha mostrado particularmente interesante en contextos universitarios. Una variante metodológica pionera en nuestro país es el Aprendizaje Basado en Proyectos Integrados (ABPI). En este contexto, se analizó la importancia que tiene la acción tutorial sobre el desarrollo de la actividad y el aprendizaje de los alumnos. Los resultados de la investigación practicada revelaron diferencias significativas en las formas de trabajar con los equipos de proyecto. En línea con resultados similares (Lafuente, 2019), encontramos que la actitud del profesorado es un elemento de importante en el afrontamiento de estas nuevas posibilidades metodológicas (Lasauskiene y Rauduvaite, 2015). Otros estudios han mostrado cómo el trabajo por proyectos mejora, por lo general, las percepciones de estudiantes y, sobre todo, de profesores (Carrió et al., 2018). Sin embargo, la diversidad de realidades ofrece también una diversidad de visiones, por parte de estos últimos. En particular, las resistencias relacionadas con el ajuste a los tiempos, procedimientos y comprensión del sistema en su conjunto dificultan la percepción de la bondad metodológica de los estudiantes, en su participación, mientras que la acción tutorial positiva, proactiva y comprometida, influyen en la percepción de la estructura, organización y evaluación de la propuesta.

Si bien, no se halló una influencia significativa en la motivación general de los alumnos hacia el trabajo por proyectos, sí se encontraron indicios relacionados con el sentido de la justicia en la adecuación de tiempos, distribución de tareas y proyectos y de elementos de evaluación, que mostraron una mayor sensibilidad en los casos en que la acción tutorial no había sido normativa.

Tomadas en conjunto, el estudio realizado, inicial en el contexto del diseño amplio planteado, se observaron dos conclusiones fundamentales: en primer lugar, que ABPI resulta ser una propuesta que mejora la percepción hacia el aprendizaje de los estudiantes de Magisterio, y que la acción tutorial, pieza clave en la organización de la propuesta, es un elemento crítico en la construcción positiva de una "cultura" de acompañamiento y de una "cultura" de aprendizaje cooperativo. 


\section{Referencias.}

Ferreiro, A. A. (2018). Aprendizaje Basado en Proyectos para el desarrollo de la Competencia Digital

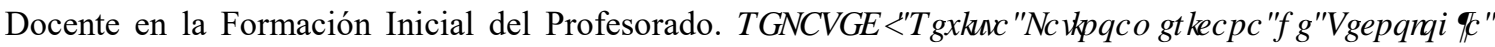
( GXFDUMD, प0 (1), 9-24. https://dialnet.unirioja.es/servlet/articulo? codigo=6566732

Arandía, M. y Fernández, I. (2012). ¿Es posible un curriculum más allá de las asignaturas? Diseño y práctica del grado de Educación Social en la Universidad del País Vasco. REDU. 5HLWDGH' RFHQFW

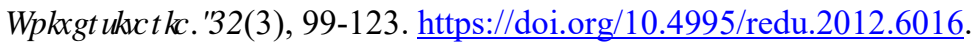

Carrió, Mar, Agell, Laia, Rodríguez, Gemma, Larramona, Pilar, Pérez, Jorge, y Baños, Josep E. (2018). Percepciones de estudiantes y docentes sobre la implementación del aprendizaje basado

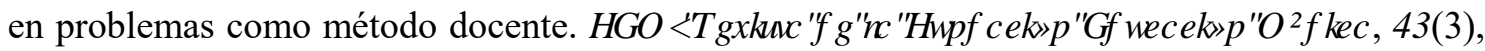
143-152. Recuperado en 11 de marzo de 2020, de http://scielo.isciii.es/scielo.php?script=sci_arttextypid=S201498322018000300006ylng=esytlng=es.

Cochran-Smith, M., Ell, F., Ludlow, L. Grudnoff, L., y Aitken, G. (2014). The Challenge and Promise of

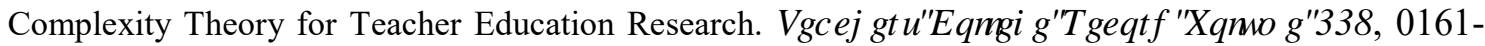
4681

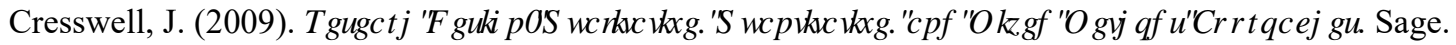

Lasauskiene, J., y Rauduvaite, A. (2015). Project-Based Learning at University: Teaching

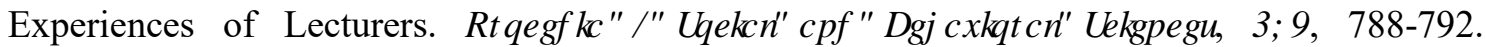
https://doi.org/10.1016/j.sbspro.2015.07.182

MacLeod, M. y van der Veen, J.T. (2019): [Scaffolding interdisciplinary project-based learning:

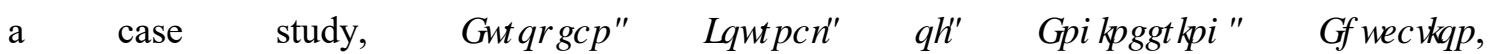
https://doi.org/10.1080/03043797.2019.1646210

Martin, S. y McQuitty, V. (2019). Complexity Theory and Teacher Education. 10.1093/acrefore/9780190264093.013.479.

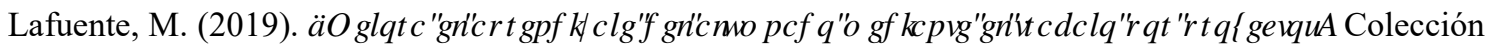
¿Qué funciona en educación? Evidencias para la mejora educativa. Institut Català de Polítiques Públiques.

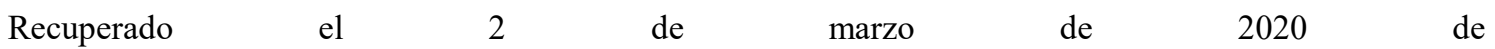
https://www.fbofill.cat/sites/default/files/Que_funciona_16 aprendizaje.pdf

Mejora de la motivación a través del trabajo en ABP, en alumnos del grado de ADE

Morales, P. T., y García, J. M. S. (2018). Aprendizaje basado en proyectos: una experiencia

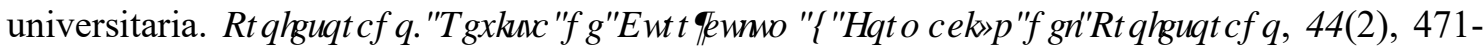
491. https://recyt.fecyt.es/index.php/profesorado/article/view/66383

Morrison, K. R. B and Tang, F. H. (2002) Testing to destruction: a problem in a small state. Assessment in Education: Principles, Policy and Practice, 9 (3), 289-317. 
Navarro, I., González, C., López, B. y Contreras, A. (2019). Aprendizaje cooperativo basado en proyectos y entornos virtuales para la formación de futuros maestros. ( GXFDUD (2), 519-541. . https://doi.org/10.5565/rev/educar.935

Olivares Olivares, S. L., López Cabrera, M. V., y Valdez-García, J. E. (2018). Aprendizaje basado en retos: Una experiencia de innovación para enfrentar problemas de salud pública. ( GXFDFyQ0 pGFD, प0, 230-237. https://doi.org/10.1016/j.edumed.2017.10.001

Pérez-Aranda, J., Molina-Gómez, J. Domínguez, L. y Rodríguez, M.C. (2015). El Aprendizaje Basado en Problemas como herramienta de motivación: reflexiones de su aplicación a estudiantes de GADE. 5HWLD GHIRLP DFIY QH, QQRYDFIy Q( GXFDMDD8 QIYHUWDUD, 8(4), 189-207.

Santonus, M. (1998) Simple, Yet Complex. http://www. cio.com/archive/enterprise/041598

Shpeizer, R. (2019). Towards a Successful Integration of Project-based Learning in Higher Education: Challenges, Technologies and Methods of Implementation. 8 QYHHWO-RXLDDORI( GXFDMRQDO5 HMDFF, 口(8), 1765-1771. https://doi.org/10.13189/ujer.2019.070815

Toledo Morales, P. y Sánchez García, J.M. (2018). Aprendizaje basado en proyectos: una experiencia universitaria. Profesorado: Revista de curriculum y formación del profesorado, 22 (2), 429-449.

Zamora Sánchez, R.; Falcón Mantilla, M. Tapia Pullas, P. (2018). Implicación del modelo de aprendizaje basado en proyectos colaborativos en el contexto universitario. Revista de Comunicación de la SEECI, 46, 01-11. doi: http://doi.org/10.15198/seeci.2018.46.01-11 